\title{
Reflexiones inspiradas en el primer párrafo del artículo primero del Código Civil peruano de 1984
}

\section{Patricia Casana de Burga}

\section{Introducción}

El primer artículo de la Constitución Política del Perú establece que el fin supremo de la sociedad y del Estado es la defensa de la persona humana y el respeto de su dignidad. Como no podía ser de otra forma, el primer párrafo del primer artículo del Código Civil está dedicado también a la persona humana. Ambos cuerpos normativos nos permiten proyectar la idea de que no se puede tocar ningún tema de derecho que sea extraño a la persona; $y$, a su vez, que existen pocos temas relativos a la persona que resultan extraños al derecho. En consecuencia, persona y derecho, o derecho y persona, son términos anclados entre sí, por lo que la definición del término persona constituye un problema perenne.

Es verdad que, a nivel teórico y formal, ya se tienen varias respuestas acerca de qué es ser persona, casi pétreas por la conocida parsimonia del derecho para recoger realidades cada vez más osadas. Sin embargo, también es cierto que los cambios vertiginosos de toda índole plantean el reto no solo de pensar, sino de repensar conceptos en apariencia inmutables. Es por esta razón que inmediatamente surgen estas interrogantes: ¿existen personas no humanas? ¿Los delfines son personas? ¿Por qué en Suecia se utiliza un pronombre neutro (hen) para quien no es ni él ni ella? ¿Se puede distinguir la vida humana en dos categorías: 
vida humana y vida verdaderamente humana? ¿Se es persona, en términos reales, a pesar de carecer de las competencias mínimas requeridas para interactuar en esta sociedad del siglo XXI? ¿Algunas personas no son en realidad solamente individuos?

Escapa a nuestros fines responder a todas esas cuestiones. Este documento de carácter exploratorio pretende dar cuenta de una reflexión en torno a la persona, punto de partida y prístina preocupación de todo aquel cuya vocación y profesión sea el derecho.

\section{2. ¿Qué es ser persona?}

Abundan las posiciones y las teorías filosóficas relativas a qué es ser persona, como las que afirman que el espíritu es el ente metafísico que otorga la categoría de persona al hombre. Sobre el particular, Torres Vásquez (2011) afirma que "en el ser humano hay una realidad material y otra espiritual que se entrecruzan indisolublemente. El ser humano es cuerpo y espíritu" (p. 10). Esta posición no kantiana considera, entonces, que el espíritu es una realidad trascendente. Existe también una noción jurídica de persona y, por cierto, posiciones teológicas, psicológicas, antropológicas y sociológicas. La cuestión, además, se aborda desde el punto de vista del origen etimológico del término y "se discute si dicho origen se encuentra en el griego, en el latín o en el etrusco" (Fernández Sessarego, 2001, p. 297).

Según el Diccionario de la lengua española, el término persona se refiere, en una acepción general, a un "individuo de la especie humana". En ese sentido ontológico, según el mismo diccionario, se trata del hombre, como "ser animado racional, varón o mujer". Cabría, entonces, preguntarnos: ¿es la razón la que nos hace humanos y es el ser humanos lo que nos hace personas?

Con relación a esta cuestión, Páez Conesa (2013) sostiene:

Tal como se utilizan de ordinario, sin embargo, las expresiones "humano" o "ser humano" son engañosamente ambiguas. A veces con estas expresiones queremos decir animal humano, esto es, un organismo de la especie del Homo sapiens. Mientras que en otros momentos queremos decir persona, esto es, un ser racional y autoconsciente. (p. 30) 
Si bien es difícil definir qué es ser persona, lo que resulta sencillo es comprender que con este vocablo se designa al sujeto en torno al cual gira el derecho. Según la Enciclopedia Jurídica Omeba (1979), persona tiene la "condición de una de las nociones básicas en el mundo de lo jurídico [y expresa] el sujeto de las relaciones jurídicas" (p. 95). Que es un concepto magno no está en duda; tampoco que "es necesario recapacitar en él, e ir considerando y reconsiderando los complejos asuntos que se ofrecen dentro de la unidad del tema" (León Barandiarán, 1962, p. 11).

No es del caso dirimir si se es persona porque el derecho así lo determina con fines instrumentales, dado que precisa de un sujeto, o si un ser humano es persona, lo reconozca el derecho o no, porque esta calidad le es intrínseca. Es verdad, sin embargo, que son posiciones contrarias y, sea que se adopte una u otra, las consecuencias podrían ser distintas. En el primer caso, bastaría con mencionar la postura de Kelsen (2011), para quien el ser humano es objeto de las ciencias naturales y la persona no es sino una noción que deriva de las normas jurídicas: "El ser humano es una realidad natural; la persona es una representación auxiliar del conocimiento jurídico, de la cual sería incluso posible prescindir. Facilita la exposición del derecho, pero no es absolutamente indispensable" (p. 76).

La postura humanista, lejos de considerar a la persona como un mero recurso técnico del derecho, la vincula con este de tal modo que los considera una realidad per se. Desde esta óptica, el ser humano tiene un sentido de justicia innato y en su relación con otros requiere un orden y rechaza la anomia; su propia naturaleza lo exige. Así, más que considerar al derecho como una estructura externa a él, lo construye desde adentro, erigiéndose en el centro de ese orden. Esta posición de raíz romana, que identifica hombre con persona, constituye una protección para el ser humano frente a intenciones totalitarias.

Independientemente de cómo definamos de manera exacta la justicia, todos -o casi todos- poseemos un sentido de justicia, que es indispensable para la cohesión de las sociedades complejas. Lo que se discute es si el sentido de justicia determina el comportamiento de las personas o si es la ley el instrumento racional de control social (Casana de Burga, 2013, p. 11). 
La ajuricidad natural es, a juicio de Hervada (2011):

[...] impensable, porque esto significaría que, por naturaleza, las relaciones de hombre a hombre no conocerían ni lo recto, ni lo justo, ni ninguna exigibilidad, ni ningún poder; sería el estado de pura anomia y de fuerza absoluta, lo cual no es propio de la condición de persona, que por naturaleza es racionalidad, exigibilidad y dominio. [...] Todo sistema jurídico positivo se basa, al menos, en la juricidad natural de los hombres, esto es, en que por naturaleza existe la capacidad y tendencia de relacionarse jurídicamente. [...] Ser persona no es de origen positivo, sino natural, porque los hombres, por naturaleza, son sujetos de derecho. (p. 119)

Por su lado, el Código Civil vigente (1984) establece que la persona humana es sujeto de derecho desde su nacimiento. Sobre la base, a contrario sensu, del aforismo según el cual no hay que distinguir donde la ley no distingue, cabría preguntarse por qué es necesario añadir el adjetivo humana al término persona, o si es posible que exista una persona no humana. En el derecho peruano, actualmente, la respuesta es contundente: no. Entonces, se podría entender que se trata de distinguir entre persona natural (o humana) y persona jurídica.

En cuanto a la pregunta de si es posible que exista una persona no humana, convendría tener presente que, en 2012, los científicos reunidos en la Asociación Estadounidense para el Avance de la Ciencia postularon que, aparte de los simios, los delfines y las ballenas son personas no humanas. Thomas I. White (2010), profesor de ética de la Universidad de Loyola Marymount, sostiene que las investigaciones sugieren que los delfines son "personas no humanas", pues está comprobado que -al igual que los humanos- son individuos únicos y autoconscientes, con personalidades diferentes, recuerdos y un sentido de sí mismos. Por si fuera poco, señala que tienen el poder de reflexionar y escoger sus acciones. Todo ello tiene grandes implicancias éticas.

Esta sugestiva posición podría cotejarse con el caso seguido en la Corte Distrital de California, en Estados Unidos, a instancia de "cinco orcas", representadas por Next Friends, People for the Ethical Treatment of Animals (PETA), contra SeaWorld Parks \& Entertainment Inc. y SeaWorld LLC. En este caso, se argumentó que las orcas estaban siendo esclavizadas al ser forzadas a vivir en tanques y actuar diariamente en los parques acuáticos de California. La pretensión era que se discuta si 
resultaba aplicable la Decimotercera Enmienda a la Constitución de Estados Unidos que abolió la esclavitud. El juez Jeffrey T. Miller (2012) amparó una moción que rechaza la admisión de la demanda por carecer de jurisdicción en razón de la materia, dado que -por lo que se expone en la resolución judicial- la citada enmienda se aplica solo a humanos, pero aclaró:

A pesar de que los demandantes carecen de base para reclamar la aplicación de la Decimotercera Enmienda, ello no implica que los animales no tienen derechos legales [cursivas añadidas]; existen varios estatutos estatales y federales que les permiten a los demandantes buscar un remedio, incluyendo en algunos casos leyes penales que castigan a quienes violen obligaciones legales que protejan a los animales. (p. 7)

Siguiendo esta línea de pensamiento, en mayo de 2013, el Gobierno de la India emitió una disposición que prohibía el establecimiento de delfinarios, dado que los delfines deben ser vistos como "personas no humanas" y como tales deben tener sus propios derechos específicos, por lo que es moralmente inaceptable mantenerlos en cautiverio para propósitos de entretenimiento. Esta posición auguraría un cataclismo en el campo del derecho occidental, cuyo nítido antropocentrismo y tendencia reaccionaria parecían no comulgar con una visión tan innovadora, tal y como lo manifiesta Páez Conesa (2013):

Al comienzo de este capítulo llamé la atención hacia el hecho de que el triunfo del antropocentrismo ha sido malo para los animales no humanos, porque esta razón de nuestro estatuto moral se enfoca en la pertenencia a una especie en lugar de en los rasgos individuales, como por ejemplo, las capacidades cognitivas. El antropocentrismo debe justificar por qué la pertenencia a nuestra especie es más importante que la pertenencia a otras. (p. 33)

Tomando en consideración el apotegma de Ulpiano: "Donde hay hombre, hay sociedad; y donde hay sociedad, hay derecho", se podría colegir que es posible la vida, en general, sin el derecho, mas no la vida humana. En tal sentido, el derecho sería uno de los pilares de la humanidad. Ahora bien, cabe la pregunta de si hay, por así decirlo, "tipos" de vida humana y, por ende, de personas. ¿Se puede hablar de vida humana -a secas- $\mathrm{y}$ de vida verdaderamente humana? 
Sería común, o cuando menos no extraño, decir que una vida, para que sea verdaderamente humana, debe ser digna. En seguimiento de esa línea, cabría reflexionar acerca de si una persona que vive una vida indigna de tal estatus es -más allá de lo que se afirme conforme a derecho- apenas un individuo. Cuando menos, parecería aceptable afirmar que si la vida de una persona no corresponde a la calidad y características propias de un ser humano, es infrahumana ${ }^{1}$.

Se podría, entonces, afirmar que todo ser humano es persona por el hecho de haber nacido vivo, pero también que algunas personas viven vidas humanas y otras viven vidas infrahumanas, sea porque carecen de ciertos bienes y servicios esenciales, o porque no son capaces de funcionar adecuadamente en la sociedad (por ejemplo, porque no entienden lo que leen o porque no saben cómo reclamar sus derechos). Por cierto, para el derecho, sea como fuere que cada ser humano viva su vida, esta siempre es valiosa.

Con relación a este punto, es preciso indicar que hay un sentido biográfico del término vida, que es el que fundamenta las posturas relativas a si una vida vale la pena de ser vivida o si lo que vale la pena es terminarla, esto es, si el agregado de cosas buenas y malas en esa vida tiene un saldo positivo o negativo. Solo los seres conscientes, por ejemplo, los seres con una mente, pueden tener vida en este sentido. Pero también existe un sentido biológico de vida, como un fenómeno caracterizado por atributos que únicamente los organismos pueden poseer, como las capacidades de adaptación al ambiente, reacción a los estímulos, sostenimiento de la propia existencia, reproducción, etc. Este es el sentido de vida en la postura antropocéntrica, según la cual la vida humana siempre es valiosa: es importante para bien, y siempre lo es, que exista un miembro vivo de nuestra especie. Afirmar que siempre vale la pena vivir las vidas humanas, que es un juicio sobre el valor personal de la vida para el organismo humano de cuya vida se trata, es ciertamente compatible con el antropocentrismo (Páez Conesa, 2013, p. 31).

1 Por ejemplo, en el inciso e del artículo 13 de la Ley 126, dictada en Puerto Rico en 2013, se establece que son condiciones de vida infrahumanas no tener servicio de agua potable, servicio de energía eléctrica, artículos de primera necesidad o vivir en condiciones de extrema pobreza. 


\section{Atributos de la persona}

Según García Toma (2008), el hombre "tiene como atributos esenciales la libertad, la racionalidad y la sociabilidad que son la raíz y el fundamento de su dignidad" (p. 25). Partiendo de esta idea, el Estado y la Sociedad, con el conjunto de leyes y principios que forman el derecho, deben promover y defender el ideal de hombres y mujeres libres y racionales que coexisten unos con otros, no egoístamente, sino en una relación de alteridad. Visto desde otra perspectiva, si un conjunto de personas coexisten en determinado ámbito dentro del cual no se respeta su dignidad, se puede decir que no hay sociedad ni hay Estado, puesto que la dignidad humana es un elemento ontológico que es condición para la existencia de ambos (García Toma, 2008, p. 25).

\subsection{La libertad}

Moreno Iturralde y Ares Mateos (2012) consideran que la libertad es una capacidad fundamental y constitutiva de la persona. La comprenden en tres sentidos: la libertad trascendental o esencial, según la cual el hombre solo se realiza como hombre si emplea su libertad; el libre arbitrio, que es la capacidad de elegir entre una cosa u otra; y la libertad moral, que es "el empleo de la libertad en cuanto que supone un contenido ético o moral" (p. 92).

Por otro lado, Jacques Maritain sostenía que el hombre era una persona plena únicamente en tanto esta y la razón dominen sus sentidos y pasiones; de otro modo, será un simple individuo, "permanecerá como un animal", esclavo incapaz de guiarse por sí mismo. Así no podría aspirar a la totalidad y sería únicamente una parte (como se citó en Novak, 1992, p. 42).

Los hombres poseen determinadas cualidades que nos mueven a llamarlos personas. Pero a lo que nosotros llamamos personas no es a estas cualidades, sino a su portador. Por lo demás, hay, como es evidente, hombres que no disponen de estas cualidades. Podría parecer, pues, que esos hombres no son personas, y no pueden invocar ningún derecho a que se los acoja como personas. Esta tesis, que tiene su origen en Locke, es la de Peter Singer y Norbert Hörster. Si las personas son los portadores individuales de una "naturaleza racional", parece que no son personas aquellos hombres que todavía no disponen, o no disponen ya, o no dispondrán nunca más, de racionalidad e intencionalidad, 
como, por ejemplo, los niños pequeños, los disminuidos profundos, o los que duermen. Si el ser persona no significa que se es un caso de un concepto o un elemento de una clase, sino que se es miembro de una comunidad de acogimiento, surge la pregunta de cómo se entra en esa comunidad. Y es natural pensar que el estatuto de persona es algo que se realiza mediante la acogida en ella (Spaemann, 2010, p. 227).

Se ha indicado ya que, para el derecho, todo ser humano es persona, sin condición alguna. Ello sería congruente con aceptar que basta con ser miembro de la especie humana -la comunidad de acogimiento de la que habla Spaemann (2010)- para gozar de tal calidad, independientemente de las cualidades que se posea. Nos preguntamos si, una vez acogidos en esa comunidad, todos sus integrantes, formalmente en igualdad de condiciones por el solo hecho de pertenecer a la misma especie, pueden gozar efectivamente de los mismos derechos con independencia de sus cualidades. La respuesta parecería negativa.

\subsection{La racionalidad}

Páez Conesa (2013), siguiendo a Finnis, sostiene que persona es aquel ser con capacidades intelectuales superiores, como la libertad, la racionalidad o la deliberación. Ahora bien, aclara que Finnis se refiere por capacidad no solo a ser efectivamente capaz de involucrarse en un acto intelectual, sino también a cuando menos tener las bases orgánicas para algún acto intelectual, aunque la persona no pueda de hecho ejecutarlo por estar dañada, "por ejemplo, por enfermedad, accidente o senilidad". Esto justificaría "el reclamo de que todos los seres humanos son personas, que sus vidas son mucho más valiosas que las de los seres no humanos $[\ldots]^{\prime \prime}$ (p. 54).

Kant (1784) expresó su fe en el hombre porque es un ser de razón, capaz de pensar libremente. Para él, quien carece de entendimiento es menor de edad y aquel que, sin carecer de entendimiento, adolece de falta de decisión y del valor de servirse de su propio entendimiento es "autoculpable" de su minoría de edad. La ilustración es la salida, o remedio, para este estado de minoría. "¡Sapere aude! ¡Ten valor de servirte de tu propio entendimiento! He aquí el lema de la ilustración" (p. 1).

Por otro lado, Novak (1992) afirma que "una persona [cursivas añadidas] es algo más que un individuo" (p. 43). Liga el concepto de persona al intelecto y a la voluntad. Una persona tiene capacidad de discernimiento, de juicio, de elección y de decisión. Agrega que "una persona es 
un individuo capaz de inquirir y elegir y, por tanto, ser a la vez libre y responsable". Interesa destacar de esta definición que la capacidad de elegir, sobre la base del intelecto, es uno de los fundamentos -si no el fundamento- de la libertad, y esta lo es de la responsabilidad. Dicho de otro modo, aquel que carece de razón no es libre, y si no lo es, tampoco es responsable (Novak, 1992, p. 43)

Si bien es cierto que el estatus de persona se adquiere formalmente -por disposición de la ley- con el nacimiento, en términos más extensos que los meramente legales ser persona implica la capacidad para responsabilizarse por los propios actos y el ser tratado de cierta manera, con respeto de sus derechos. Estos dos aspectos pueden ser analizados por separado. El primero, de índole moral, requiere que el agente sea racional, que se le pueda atribuir intencionalidad o conciencia de sus actos, que tenga la capacidad de ser recíproco y de comunicarse con otros, así como que sea consciente de sí mismo. Este último requisito se entiende como un proceso mental reflexivo de orden superior (Gallagher, 2007, p. 200).

En tal sentido, Moreno Iturralde y Ares Mateos (2012) afirman que "el hombre para vivir necesita razonar. [El pensamiento es primero y por eso] podemos decir que el hombre tiene manos porque piensa. [Y agregan que] la condición de persona es aplicable al ser humano porque tiene inteligencia reflexiva y libertad moral" (pp. 21-49). Los poderes de autodesplazamiento, percepción y sensación, y el apetito son compartidos con otros animales. Lo que es distintivo de la humanidad y lo que caracteriza a la mente son los poderes del intelecto, de la razón y de la libertad racional (Hacker, 2013, p. 27).

Contrariamente a lo expuesto hasta aquí, para Blasi (2009), la racionalidad no es constitutiva de la persona, pues si lo fuera, no todo ser humano sería persona o no lo sería en todo momento, pues sus atributos -entre ellos la razón- cambian en el transcurso de cada vida humana. Además, como los atributos humanos son poseídos en distintos grados, según los individuos y las diferentes circunstancias individuales, habría entonces distintas categorías de personas [cursivas añadidas]. Esta perspectiva sería compatible con la noción jurídica contemporánea de persona en el derecho occidental moderno, que descarta la concepción estamental de persona, a diferencia de aquella del derecho romano que solo reconocía como persona a quienes gozaban de los tres estatus que los dotaban de plena capacidad jurídica (el estado de libertad, el estado de ciudadanía y el estado de familia) (Blasi, 2009, pp. 109-110). 
Como es sabido, en el derecho romano imperial, algunos seres humanos eran considerados jurídicamente cosas. Inclusive en el siglo XVI cabía preguntarse si los aborígenes del Nuevo Mundo eran personas. En 1537, Paulo III declaró en la bula Sublimus Dei que los indios son verdaderos hombres: "We $[\ldots]$ consider, however, that the Indians are truly men $[\ldots]^{\prime \prime}$.

En el siglo XVIII, la situación persistía. El caso del esclavo James Somerset sirve para graficarla. Fue capturado en África alrededor de 1749 y trasladado a Estados Unidos de América. Dos décadas después, su dueño lo llevó a Inglaterra en donde escapó. Al ser recapturado, fue encarcelado. En su nombre se interpuso una demanda histórica pidiendo su libertad. El abogado de Somerset logró una "transubstanciación legal" de su defendido de cosa en persona al convencer al juez de que sobre la base del Common Law la esclavitud humana es odiosa.

\subsection{La sociabilidad}

Si nos preguntamos qué es vida humana, podríamos responder desde diversas posiciones, como la antropológica, la biológica o la religiosa. Independientemente de la perspectiva, se podría intuir, con carácter "supracultural", que la vida humana es la vida en sociedad.

Queramos o no, nos hallamos inclinados o abocados a la convivencia, por mucho que nos hagamos la ilusión de creernos dioses autosuficientes, o una parte más de la naturaleza. $\mathrm{O}$, rizando el rizo, ambas cosas a la vez, tal como sucede con la parafernalia New Age, que apunta al equilibrio con uno mismo y con el cosmos, aunque sin pasar, sospechosamente, por la dimensión social y política (Saborit, 2006, p. 19).

Ya el Estagirita dijo que el hombre es infinitamente más sociable que todos los animales que viven en grey: "Y el que no puede vivir en sociedad, o no necesita nada por su propia suficiencia, no es miembro de la ciudad, sino como una bestia o un dios". Él contempla al hombre como una parte de un todo mayor, que es la ciudad (la que para el caso representa a la sociedad). Entonces, su lógica va del más al menos. Primero es el conjunto y luego la parte. Solo dentro de ese todo, el ser humano puede cumplir su función.

En cuanto a este punto, nos formulamos las preguntas que Condorcet (1988) se hacía en 1793: 
¿Cómo, en esta asombrosa variedad de trabajos y productos, de necesidades y recursos, en esta aterradora complicación de intereses, que enlazan la subsistencia aislada y el bienestar del individuo al sistema general de la sociedad, que lo hace dependiente de todos los accidentes de la naturaleza y de cada suceso político, lo que, como es, incrementa su capacidad de experimentar el gozo o la privación al globo entero, cómo, en este aparente caos, vemos sin embargo, a través de una ley general del mundo moral, que los esfuerzos que cada uno hace por sí mismo sirven al bienestar de todos y, a pesar de la aparente colisión de intereses contrapuestos, el interés común requiere que cada uno sepa cómo interpretar su propio interés y sea capaz de alcanzarlo sin obstrucción? [cursivas añadidas]. (p. 219)

No obstante lo indicado, es verdad que el ser humano también requiere de una esfera de intimidad, lejos del otro. Necesita recogerse, esto es, según el Diccionario de la lengua española, "retirarse a algún sitio, apartándose del trato con la gente". Además, la postura filosófica y social predominante del individualismo occidental determina que los seres humanos, en general, tengan como meta la autosuficiencia en búsqueda de los mayores grados de independencia. Se rehúye la responsabilidad y el riesgo que supone establecer una relación plena con otro ser humano para no poner en peligro nuestra ilusión contemporánea de autosuficiencia. En todo caso, al otro se le acepta, pero solo tras moldearlo y adaptarlo a nuestros intereses. Si el café se toma sin cafeína y la cerveza sin alcohol, al otro únicamente se le tolera sin verdadera alteridad (el fenómeno del "turismo sexual" constituye su manifestación más extrema y lamentable) (Supiot, 2007, p. 15).

La tesis orteguiana: "Yo soy yo y mi circunstancia y si no la salvo a ella no me salvo yo" parecería referir tal "circunstancia" a lo experimentado, es decir, a lo pasado, como seres en diaria formación acumulativa. Desde otra perspectiva, implicaría que no somos total o verdaderamente en soledad con nuestro yo, sino en medio, al lado, entre, con o sobre "algo" (la circunstancia); es en esa circunstancia que existimos porque coexistimos.

La subjetividad sin intersubjetividad es imposible. Una de las equivocaciones de la filosofía moderna es justamente que, al llegar a la relación del sujeto, la intersubjetividad es un tema que no ha podido afrontar. El que se ha preocupado a fondo por esta cuestión es Husserl, pero me parece que la solución del padre de la fenomenología no es suficiente, 
pues no acaba de ver en qué estriba la intersubjetividad, es decir, en qué estriba la persona en su relación con otras (Polo, 2011, pp. 145-146).

Dado que el ser humano es sociable, se comprende que alcance su plenitud en comunidad, desde la reducida comunidad del ámbito familiar o doméstico hasta la política, en la que ejerce su ciudadanía. Como sostiene Goleman (2006), estamos "cableados" para sociabilizar y tenemos un sesgo hacia la empatía, la cooperación y el altruismo, siempre y cuando desarrollemos nuestra inteligencia social (p. 20).

Portocarrero (2013), por su parte, afirma que el neoliberalismo, con su culto al éxito individual y al consumo, ha debilitado los vínculos sociales, erosionando los valores colectivos que fundamentan la moralidad pública y la vigencia de la ley. El máximo valor de la vida sería el éxito y quien no entre en un ranking será un fracasado (p. A32). “De esta manera la gente que tiene menos oportunidades queda reducida al silencio, pues se insiste en que si no logra el ansiado éxito la culpa es solamente suya" (Portocarrero, 2013, p. A32).

\section{La dignidad}

En lo atinente a la dignidad humana, hay consenso en su importancia primordial, pero no nos consta que haya un acuerdo sobre el contenido concreto y aplicable de esta noción capital. En una primera aproximación, parece que ser digno es comportarse de acuerdo con lo que uno es: una persona humana. Por lo tanto, dignidad parece referirse a la coherencia con el propio ser (Moreno Iturralde y Ares Mateos, 2012, p. 52).

El Tribunal Constitucional peruano, en el Caso N. ${ }^{\circ}$ 10087-2005-PA, caracterizó a la dignidad de la persona humana como "un dínamo de los derechos fundamentales; por ello es parámetro fundamental de la actividad del Estado y de la sociedad, así como la fuente de los derechos fundamentales". El mismo Tribunal, en el Caso N. ${ }^{\circ}$ 02273-2005-HC, dejó en claro que la realización de la dignidad humana no se satisface "en la mera técnica de positivización o declaración por el derecho", sino que se debe garantizar el goce de garantías y "niveles adecuados de protección a su ejercicio". Además, que la dignidad humana es "comprehensiva enunciativamente de la autonomía, libertad e igualdad humana" y que todas ellas son "necesidades humanas que emergen de la experiencia concreta de la vida práctica". Es decir, si una persona no goza efectiva, y no solo declarativamente, de los derechos a la autonomía, libertad e igualdad en su vida práctica, no vive 
una vida digna. Y si esto es así, no está en posición de gozar de los demás derechos fundamentales.

Existe una gran preocupación por la persona y sus derechos subjetivos, siempre la hubo, pero a diferencia de lo que acontecía en "siglos anteriores donde la protección de la propiedad era la principal preocupación del derecho" (Fernández Sessarego, 2001, p. 290), hoy se incide en la protección de aquellos derechos que se consideran una proyección del hombre como el derecho a la libertad, a la igualdad, a la intimidad, a la salud, a la propia imagen, a la voz, entre otros, pero también -por qué no- el derecho al arte (o a disfrutar del arte), el derecho a la belleza o el derecho a la tranquilidad. Nuestra weltanschauung demanda la irreductibilidad de cuando menos un núcleo de derechos y su inviolabilidad como algo "coesencial" a la persona.

\section{La igualdad}

Los seres humanos somos únicos y diferentes; intuitivamente podríamos decir, con carácter general, que la idea de que somos iguales no es verdad. Las diferencias son irrefutables y saltan a la vista. Esa heterogeneidad es una realidad. Sin embargo, modernamente se busca el ideal de la igualdad -concepto en ebullición-, por la que hay una "casi universal adoración" (Rothbard, 1995). En concordancia, la desigualdad es repudiada y se le asocia con el elitismo y la exclusión social, tanto como se propugna la masificación y la inclusión social. Más aún, se acusa a la desigualdad de ser el origen de los "movimientos sociales y populares" y "no importa cuánto se busque mirarla críticamente, la desigualdad tendrá siempre un sentido peyorativo, una clara asociación con el mal" (Cuenca, 2013, p. 35).

Si nuestra propia identidad emerge de la diferencia con relación a otro, si no podemos sostener nuestra identidad en total aislamiento, si la igualdad es inalcanzable, estamos compelidos a aceptar la identidad del otro como tal, aunque sea porque, en primer lugar, es la fuente de nuestra propia identidad (Adib-Moghaddam, 2012, p. 36).

Según Humala, la desigualdad es el origen de la violencia. Cuando aquella aumenta, se genera resentimiento y de ahí a la violencia solo hay un paso: "La desesperación, fundada en la pobreza -personificada en Jean Valjean de Los miserables-, conduce inevitablemente a un camino distinto al democrático, a uno caótico y violentista, añade nuestro man- 
datario" (como se citó en Meléndez, 2014, p. 14). Sin embargo, Meléndez (2014) se opone a esta opinión:

Se ha demostrado largamente que la violencia política no está asociada exclusivamente a clases bajas. [...] Insistir, en pleno siglo XXI, en este argumento desactualizado solo lleva a reproducir estereotipos sociales en un país desigual, clasista y discriminativo. La gravedad del asunto recae en la institucionalización de la malversada idea-fuerza de que el pobre es un radical en potencia, lo que recalca la archimanida discriminación histórica. (p. 14)

Desde la Ilustración, Rousseau (1987) rechazó el concepto de desigualdad natural, con lo que planteó una de las ideas políticas que sirvieron de inspiración a la Revolución francesa. A este filósofo se le atribuye el haber sentado "algunas de las más sólidas ideas ilustradas sobre la desigualdad [...], que la igualdad entre los hombres es un hecho natural y que la ruptura de ese orden natural es, entonces, un asunto político; por lo tanto, el Estado debería estar en capacidad de atender los derechos naturales de los individuos" (Cuenca, 2011, p. 32). Sea o no de índole natural, cuestión que no es del caso dirimir en este documento, lo cierto es que se habla de desigualdad económica, social, de género, entre otras "antiguas desigualdades [que] coexisten con otras nuevas formas aún más complejas" (Cuenca, 2011, p. 31).

En nombre de la igualdad, se establecen políticas de Estado o se diseñan camas de Procusto. Hasta parece políticamente incorrecto abogar por las diferencias a las que hay que combatir, eliminar, atacar o-si ello no es posible- amenguar y "sufrir". Todo ello se comprende si tomamos en cuenta que apenas en el siglo XX el Holocausto nazi se fundó en la alegada superioridad de una raza sobre otras y el no derecho a vivir de algunos "seres inferiores" como, por ejemplo, los homosexuales. En oposición a ello, o quizás en respuesta, el primer párrafo del preámbulo de la Declaración Universal de Derechos Humanos (1948) sostiene:

[...] la libertad, la justicia y la paz en el mundo tienen por base el reconocimiento de la dignidad intrínseca y de los derechos iguales e inalienables de todos los miembros de la familia humana. [Más concretamente, en el artículo 6 se reconoce que] todo ser humano tiene derecho, en todas partes, al reconocimiento de su personalidad jurídica. 
Es decir que -jurídicamente- por el solo hecho de ser humano se es digno y se posee derechos iguales e inalienables, además del derecho al reconocimiento de la personalidad jurídica.

Una visión mucho más general identifica el atributo de pertenecer a la raza humana como el que nos da las razones decisivas para un trato fundamentalmente igualitario de todos los seres humanos, sin que tenga ninguna vinculación con sus atributos individuales. Creo que esta postura está equivocada, pero no pretendo deslegitimar a quienes la defendieron en el pasado y todavía se adhieren a ella hoy en día. Probablemente fue para bien de todos nosotros que se propagó la convicción de que todos los seres humanos son fundamentalmente iguales, dado que desplazó los puntos de vista equivocados sobre lo que constituyen bases sólidas -raza, género, ascendencia y así sucesivamente- para un trato fundamentalmente desigual de grupos de personas. Sin embargo, fue malo para otros seres conscientes. Dado que pertenecen a otras especies, se justificó que nuestro trato hacia ellos sea moralmente menos demandante, también con independencia de sus características individuales (Páez Conesa, 2013, p. 27).

¿Todas las personas son iguales? ¿Es deseable la igualdad? ¿De qué igualdad hablamos? Para Rothbard (1995), "[...] al impulso igualitario, una vez concedida legitimidad, no se le puede apaciguar". Agrega que en contraposición al igualitarismo "clásico" se yergue un igualitarismo grupal o nuevo igualitarismo, "que enfatiza no que cada individuo debe ser igual, sino que los ingresos, el prestigio o el estatus de una aparentemente inacabable variedad de 'grupos' deben ser hechos iguales entre sí". Ese nuevo igualitarismo sería el caballito de batalla del que se sirve la élite (intelectuales, académicos, formadores de opinión, periodistas, burócratas, abogados, etc.), los "ricos", en pro de los "pobres". Los "ricos" neoigualitarios buscan grupos "oprimidos" -antes había un solo grupo "oprimido" que era el proletariado- a los cuales defender, y esto, por las razones que expone Rothbard (1995), los favorece aumentando su poder político. "Comenzó con los oprimidos negros y luego, en rápida sucesión, había mujeres, hispanos, indígenas, inmigrantes, inválidos, los jóvenes, los viejos, los enanos, los muy altos, los gordos, los sordos, y así sucesivamente ad infinitum" (Rothbard, 1995).

Las reflexiones de Rothbard (1995) traen a colación la utilización del término caviar para designar a ciertos grupos que, a pesar de provenir de clases sociales acomodadas, pregonan posiciones de izquierda. No es 
infrecuente acusar a la gauche caviar, gauche champagne, toscana-zosi o radical chic de utilizar para sus propios fines y como bandera, o prácticamente como fachada, los reclamos de grupos oprimidos, abusados, excluidos, discriminados o cualquier otro adjetivo que los coloque al margen del "sistema" (económico, social, político o cultural).

Toda nuestra educación, nuestro desarrollo científico, nuestro amor por la belleza, nuestras ocupaciones, presuponen un ambiente constantemente limpiado y atendido por otros, preparado por otros, el trabajo de alguien es esencial para proveernos a nosotros del tiempo libre necesario para nuestro desarrollo mental [cursivas añadidas], ese tiempo libre, esa ociosidad activa que le permite a un pensador concentrarse, a un poeta soñar, a un epicúreo disfrutar de sí mismo, lo que alimenta el desarrollo rico, lujoso, caprichoso y poético de nuestras personalidades aristocráticas (Herzen, 2011, p. 63).

Como no puede ser de otra manera, hay un punto de vista jurídico en relación con el derecho a la igualdad, el cual es reconocido en la Declaración Universal de Derechos Humanos, la Declaración Americana de los Derechos y Deberes del Hombre, el Pacto Internacional de los Derechos Civiles y Políticos en la Convención Americana sobre Derechos Humanos. En el Perú, la Constitución establece en su artículo 2, inciso 2, que toda persona tiene el derecho fundamental a la igualdad ante la ley y que nadie debe ser discriminado por motivo de origen, raza, sexo, idioma, religión, opinión, condición económica o de cualquiera otra índole. El artículo 26, inciso 1, de la misma Carta consagra el principio de igualdad de oportunidades sin discriminación en la relación laboral.

Nótese que la igualdad que proclama la Constitución es "ante la ley", la cual tiene como uno de sus atributos el ser general y abstracta, amén de obligatoria con carácter universal. De este modo, se podría entender que "el principio de igualdad quedó subsumido dentro del principio de legalidad" (García Toma, 2008, p. 103) y que, en su concepción, se podría satisfacer de manera formal. Actualmente, ha quedado claro que la igualdad, para que sea tal, no puede ser solo retórica, sino que debe expresarse en la vida diaria de las personas como una realidad que el Estado debe garantizar.

El derecho a la igualdad parte de la premisa según la cual todos somos iguales en cuanto todos somos seres humanos. Esa es una igualdad esencial, en oposición a nuestras diferencias, que son cuestiones accidentales. La igualdad no implica que todos tengan exactamente los 
mismos derechos y obligaciones, pues "el tratamiento jurídico de las personas debe ser igual, salvo en lo atinente a la diferencia de sus cualidades accidentales y de la naturaleza de las cosas que los vinculan coexistencialmente" (García Toma, 2008, p. 110). En efecto, el sistema jurídico se construye sobre la base del reconocimiento de la autonomía de la persona y de la atribución de responsabilidad. Las personas, autónomas y libres, son en esencia iguales para el derecho, más allá de sus diferencias reales. De esa igualdad formal derivaría que el derecho se debe construir, como regla, sobre la base de normas generales y universales, y solo como excepción admitir la incorporación de leyes especiales, las cuales, a tenor del artículo 103 de la Constitución, pueden expedirse porque así lo exige la naturaleza de las cosas, pero no por razón de las diferencias de las personas.

Lo cierto es que, frente a la igualdad formal del derecho, nos encontramos con la desigualdad real expuesta a cada paso de la vida diaria y, lo que es más, con una desigualdad cada vez "más desigual". Ello impulsa a la proliferación de legislación específica para ciertos grupos sensibles (ancianos, adolescentes, mujeres, niños, niñas, discapacitados, migrantes, analfabetas, entre otros) o para grupos minoritarios (como sería el caso, por ejemplo, de la unión civil para personas del mismo sexo), con lo cual se reconoce implícitamente que la universalidad del derecho no es un dogma. Por ello, es importante tener presente que el derecho "ocupa una parcela de la vida social del hombre. Los juristas necesitamos reconocerlo [cursivas añadidas] con modestia, si no queremos perder el sentido de la proporción e incluso el de la realidad" (Marín Pérez, 2010, p. 26).

\section{La persona-ciudadano}

Un individuo -el ser humano vivo en su estado de naturaleza- se forma como (¿o transforma en?) ciudadano mediante un proceso socio-histórico en el cual la educación juega un rol gravitante.

El concepto de formación alude a un proceso en el cual algo o alguien adquiere una determinada forma. Este proceso de tomar forma puede entenderse como un desarrollo evolutivo desde un punto inicial inmanente, que es definido como informe y no desplegado en sus posibilidades, o bien puede entenderse como una in-formación, cuyo punto inicial está fuera, en alguna instancia in-formativa, es decir, en formas capaces de ir hacia lo que no tiene forma y moldearlo de alguna manera. En otras palabras, en la idea de formación se ponen en juego 
factores endógenos y exógenos. Por eso es tan tentador usar este término para hablar de educación (Cullen, 2007, p. 51).

Se trata de formar o construir una "ciudadanía activa [...], que supone una autonomía de palabra, una movilización cultural entre los diferentes niveles de implicación (territorial, organizacional, gubernamental, institucional)" (Dos Santos, 2010, p. 200). Esta preocupación se inserta "en una corriente internacional [cursivas añadidas] preocupada por cultivar la educación para la ciudadanía democrática (ECD) como vía de mejora y regeneración de las democracias" (Naval Duarte y Ugarte Artal, 2007, p. 88).

A la luz de lo anterior, me atrevo a decir que en esta compleja sociedad del conocimiento -con múltiples formas de producción y distribución del poder en relaciones matizadas, cuando no impregnadas por múltiples culturas coexistentes-, para ser personas-ciudadanos, y no solo individuos, se requiere acceder (en la práctica y no formalmente) a educación de calidad. De otro modo, con una pseudoeducación, solo en apariencia se nos abriría el camino hacia la iluminación, al desarrollo del intelecto y al ejercicio en el uso del propio entendimiento. Si esto es así, veremos mermada nuestra capacidad de decisión y de elección y, por ende, no seremos libres. Quien no accede a educación de calidad se mueve en la limitada esfera del analfabetismo funcional y es incapaz, en detrimento personal y de toda la sociedad, de asumir los retos que el siglo XXI plantea, tanto en términos de oportunidades como de desafíos.

La creciente preocupación por la educación ciudadana revela una toma de conciencia acerca de que la fortaleza o debilidad de una democracia depende, en buena medida, de los conocimientos, competencias y disposiciones de sus ciudadanos en relación con la vida en común. "Resurgen diversas reflexiones y propuestas sobre cómo educar para la ciudadanía a las nuevas generaciones como condición para mejorar nuestra convivencia social" (Fascioli Álvarez, 2012, pp. 35-36).

¿Qué rol juega el lenguaje en la formación de la persona? En el prólogo del Evangelio de Juan se aprecia el rol supremo de la palabra: Dios es la palabra y la palabra es el origen. Sin esta no hay nada. Del mismo modo, el orden, en general, y el derecho, en particular, no son posibles sin la palabra. Vincent-Thomas (2007) cita al sabio dogón Ogotemmeli, quien afirma que estar desnudo es estar sin habla (p. 4). Agrega el mismo autor que el ser sin habla es parcialmente un ser muerto (Vincent-Thomas, 2007, p. 4). 
Según Moreno Iturralde y Ares Mateos (2012), el lenguaje es exclusivamente humano; el hombre habla porque tiene inteligencia. Sobre el particular, Hacker (2013) afirma que una criatura que tiene una mente es una criatura que puede razonar $y$, por ende, ser sensible a las razones (p. 117). Añade que ser sensible a las razones y tener la habilidad de razonar (hacer inferencias) son la prerrogativa de los animales que usan el lenguaje (Hacker, 2013, p. 30).

Las personas tienen características que les dan identidad, las cuales son necesarias, irrenunciables y únicas; estas son el nombre, el domicilio, la nacionalidad, la capacidad, el patrimonio y el estado civil ${ }^{2}$. La capacidad es la aptitud que tienen las personas físicas para ser sujetos de relaciones jurídicas (activos y pasivos).

El hombre es un animal metafísico. Como ser biológico, aborda el mundo a través de sus órganos sensoriales.

Sin embargo, su vida se despliega no solamente en el universo de las cosas, sino también en un universo de signos. Dicho universo se extiende, más allá del lenguaje, a todo aquello que materializa una idea y hace así presente en la mente lo que físicamente está ausente. [...] Enlazar una significación consigo mismo y con el mundo resulta vital para no caer en el sinsentido, es decir, para volverse un ser racional y seguirlo siendo. (Supiot, 2007, p. 9)

Según Supiot (2007), la lengua materna es el primero de los recursos dogmáticos indispensables para la constitución del sujeto. Por medio de la palabra, el sujeto adquiere conciencia de su ser y desde su nacimiento, cuando aún no ha accedido a la palabra, es nombrado e inscrito en una filiación. Es decir, antes de poder decir yo:

[...] la ley ha hecho de cada uno de nosotros un sujeto de derecho. Son los lazos del derecho y de la palabra los que hacen que cada recién nacido acceda a la humanidad. Y el ser humano privado de vínculo con sus semejantes está destinado a la idiocia [cursivas añadi-

2 Respecto al estado civil, por ahora cuando menos, son los siguientes: soltero o soltera, casado o casada, viudo o viuda y divorciado o divorciada. La discusión reciente vinculada a la unión civil no matrimonial para personas del mismo sexo plantea la inclusión de un estado civil adicional que sería el de "compañeros civiles". 
das], tanto como lo está quien permanece encerrado en su propia visión del mundo y es incapaz de comprender que hay otras visiones posibles. Así, la "aspiración a la justicia" representa un dato antropológico fundamental. (Supiot, 2007, pp. 10-11)

Cabría reflexionar acerca de la dificultad que plantea en un país plurilingüe como el Perú que el derecho, fundamentalmente escrito y solo en castellano, sea obligatorio para todos en la República. Por si fuera poco, ese derecho está escrito en un lenguaje rebuscado, afectado, anacrónico y, por eso y más, abstruso. "En las sociedades modernas con grandes cantidades de derecho escrito y sistemas legales complejos, es necesario ir más allá del alfabetismo básico para entender y utilizar la ley" (Athabasca University, 2011).

Lo anterior cobra particular relevancia si tomamos en cuenta que el derecho es una herramienta imprescindible para una "vida significativa y activa en nuestra cultura cada vez más legalista y litigiosa" (Athabasca University, 2011).

[...] cuando reunimos a un grupo de escritores mediocres en un área compleja con su propia jerga atasca-mentes, plagada de expresiones afectadas que desplazan a las palabras comunes, el resultado es lo suficientemente predecible. Pero hay algo aún peor: haga que los estudiantes de derecho examinen rumas de rumas de prosa tediosa, hiperformal y anquilosada. Acultúrelos en la pomposidad. ¿Qué se supone que obtendrá? Terminará con un escritor de textos legales promedio: que palabrea, anticuado, artificial y usualmente agramatical. (Garner, 2001, p. xvii)

Según el Instituto Nacional de Estadística e Informática (INEI), la tasa de analfabetismo a nivel nacional estimada al año 2012 es de 6,2 \%. Frente a ese dato, tenemos los resultados del Programme for International Student Assessment (PISA, 2012). Resulta que nuestro país ocupa el último lugar en lectura, prueba que se enfoca en la habilidad para usar la información escrita en situaciones de la vida real (OECD, 2012, p. 19). El alfabetismo en lectura consiste en "comprender, usar, reflexionar sobre y servirse de textos escritos a fin de alcanzar sus propias metas, desarrollar su propio conocimiento y participar en la sociedad" (OECD, 1999, p. 20).

Entonces, solo el 6,2 \% de los peruanos es analfabeto, pero muchos de los que saben leer no pueden usar la información escrita como herra- 
mienta cognitiva o para participar en la sociedad. Esto es, son analfabetos funcionales. $\mathrm{Si}$ a esto le añadimos que, en particular, el lenguaje legal es muy rebuscado, cae por su propio peso que es difícil, cuando no imposible, que el grueso de las personas conozca y comprenda las cuestiones de derecho que, quiéranlo o no, les atañen de forma directa en prácticamente todas las esferas de sus vidas.

La persona es un ser complejo. No sabemos en qué medida es cuerpo y en qué medida es espíritu, ¿o acaso es solo cuerpo? No es un mero individuo luchando por su supervivencia. Es, en primer lugar, humana y, además, es sujeto de derecho. Se le adhieren atributos, pero el mundo le exige resultados que no se sustentan solo en ellos. Existe y coexiste. Sus posibilidades de "éxito" en una sociedad hiperindividualista - por incongruente que parezca- dependen de una serie de factores, como el elemental poder que confiere dominar el castellano, comprender lo que se lee, acceder a la justicia, contar con un mínimo de recursos económicos, ser competente, acceder a redes de diverso tipo, entre una miríada de exigencias que de no ser satisfechas generan exclusión social. Sea como fuere, incluida o excluida del entramado social, la muerte pone fin a la persona.

\section{Referencias}

Adib-Moghaddam, A. (2012). What is the Clash of Civilizations? Contrapuntal Reflections. En M. Mahdavi y W. A. Knight (Eds.). Towards the Dignity of Difference? Neither End of History nor Clash of Civilization (pp. 27-42). Abingdon, Reino Unido: Ashgate Publishing Group. Recuperado de http://site.ebrary.com/lib/bibudlima/ Doc?id=10583430\&ppg=65

Athabasca University. (2011). What is legal literacy? Recuperado de http://www.athabascau.ca/syllabi/lgst/docs/LGST249_sample.pdf

Blasi, G. F. (2009). ¿Cuál es el estatus jurídico del embrión humano? Un estudio multidisciplinario. En C. A. Calderón Puertas, M. E. Zapata Jaén, y C. Agurto Gonzales (Coords.). Persona, derecho y libertad. Nuevas perspectivas. Escritos en homenaje al profesor Carlos Fernández Sessarego. Lima, Perú: Motivensa, Editora Jurídica.

Casana de Burga, P. (2013). Las competencias directivas del juez supremo peruano. Lima, Perú: Universidad de Lima, Fondo Editorial. 
Condorcet, M.-J.-A. N. C. (1988). Esquisse d'un tableau historique des progrès de l'espirit humaine. París, Francia: Garnier-Flammarion.

Cuenca, R. (2011). Argumentos filosóficos sobre la desigualdad. En J. Cotler y R. Cuenca (Eds.). Las desigualdades en el Perú: balances críticos. Lima, Perú: Instituto de Estudios Peruanos.

Cullen, C. A. (2007). ¿Patriotas o cosmopolitas? Los dilemas de la intersubjetividad ciudadana. Metapolítica, 11(53), 50-56.

Dos Santos, J. (2010). Da cidadania activa: o sincretismo do sujeito racional e do homem relacional. Signo y pensamiento, 29(56), 194-205.

Enciclopedia Jurídica Omeba (tomo XXII). (1979). Buenos Aires, Argentina: Bibliográfica Argentina.

Fascioli Álvarez, A. C. (2012). Una educación ciudadana más allá de los derechos. Andamios, 9(18), 35-57.

Fernández Sessarego, C. (2001). ¿Qué es ser persona para el derecho? Derecho PUC, 54, 289-333.

Gallagher, S. (2007). Moral Agency, Self-Consciousness, and Practical Wisdom. Journal of Consciousness Studies, 14(5-6), 199-223. Recuperado de http://ummoss.org/gallagher07jcs*.pdf

García Toma, V. (2008). Los derechos fundamentales en el Perú (1. a ed.). Lima, Perú: Jurista Editores.

Garner, B. A. (2001). Legal Writing in Plain English. Chicago: University of Chicago Press.

Goleman, D. (2006). Inteligencia social: la nueva ciencia para mejorar las relaciones humanas. México: Planeta.

Hacker, P. M. S. (2013). Intellectual Powers: A Study of Human Nature. Somerset, Reino Unido: Wiley. Recuperado de http://site.ebrary. com/lib/bibudlima/Doc?id=10731778\&ppg=17

Hervada, J. (2011). Introducción crítica al derecho natural (11. ${ }^{\mathrm{a}}$ ed.). Pamplona, España: Ediciones Universidad de Navarra.

Herzen, A. (2011). From the Other Shore y The Russian People and Socialism. Londres, Reino Unido: William Clowes and Sons, Limited. Recuperado de http://altheim.com/lit/herzen-ftos.html

Instituto Nacional de Estadística e Informática. (2012). Tasa de analfabetismo de la población de 15 y más años, según departamento, 2005-2012. Recuperado de www.inei.gob.pe/media/MenuRecursivo/Cap05050.xls 
Kant, I. (1784). Respuesta a la pregunta: ¿Qué es la ilustración? Recuperado de http://geografiaunal.files.wordpress. com/2013/01/kant_ilustracion.pdf

Kelsen, H. (2011). Teoría pura del derecho. Introducción a los problemas de la ciencia jurídica. Madrid, España: Trotta.

León Barandiarán, J. (1962). Prólogo. En C. Fernández Sessarego. La noción jurídica de persona. Lima, Perú: Universidad Nacional Mayor de San Marcos.

Ley 126, Ley sobre el Protocolo de Servicios Interagenciales para las Personas de Edad Avanzada de Puerto Rico que viven en Condiciones Infrahumanas. Puerto Rico, 31 de octubre de 2013. Recuperado de http://www.lexjuris.com/lexlex/Leyes2013/ lexl2013126.htm

Marín Pérez, P. (1979). Reglas de conducta y derecho. En Introducción a la ciencia del derecho (pp. 25-47, 4. ${ }^{\mathrm{a}}$ ed.). Madrid: Tecnos.

Meléndez, C. (8 de mayo del 2014). Pensamiento Humala [Columna de opinión]. Perú21, p. 14.

Miller, J. T. (2012). Corte distrital de los Estados Unidos de América. Distrito de California. Sentencia expedida en el Caso N.o 11cv2476 JM (WMC). Recuperado de http://www.earthintransition.org/wp-content/uploads/2012/02/Court-ruling-in-PETA-v-SeaWorld.pdf

Moreno Iturralde, J. I., y Ares Mateos, G. (2012). La dignidad de todo ser humano (1. ${ }^{\mathrm{a}}$ ed.). Madrid, España: Ediciones Internacionales Universitarias.

Naval Duarte, C., y Ugarte Artal, C. (2007). Educación para la ciudadanía y los derechos humanos, concepto clave en la nueva ley de educación española. Revista Panamericana de Pedagogía: Saberes y Quehaceres del Pegagogo, 11, 77-91.

Novak, M. (1992). Personas libres y el bien común (1. ${ }^{\text {a }}$ ed.). México: Editorial Diana.

Organisation for Economic Co-operation and Development (OECD). (2012). Estadística OEDC. Recuperado de http://www.oecd.org/

Organisation for Economic Co-operation and Development (OECD). (1999). Estadística OEDC. Recuperado de http://www.oecd.org/

Ortega y Gasset, J. (1963). Meditaciones del Quijote e ideas sobre la novela. Madrid, España: Revista de Occidente. 
Páez Conesa, J. E. (2013). Abortion: What We Ought to Believe. An Ontological and Normative Analysis. (Tesis para optar el grado de doctor). Universidad Pompeu Fabra, Barcelona.

Papa Paulo III. (1537). Sublimus Dei [Bula]. Recuperado de http://www. papalencyclicals.net/Paul03/p3subli.htm

Planas, E. (21 de enero de 2014). Vik Muñiz: “Vivimos en medio de un basural de información" [Entrevista]. El Comercio. Recuperado de http://elcomercio.pe/luces/arte/vik-muniz-vivimos-medio-basural-informacion-noticia-1704103

Polo, L. (2011). La esencia del hombre (1. ${ }^{\text {a }}$ ed.). Navarra, España: Ediciones Universidad de Navarra.

Portocarrero, G. (10 de agosto de 2013). Balance del neoliberalismo. El Comercio, p. A32.

Rothbard, M. N. (1995). Igualitarismo y las élites. Review of Australian Economics, 8(2), 39-57. Recuperado de http://llvimg.net/media/ blogs/index/doc/MNR-igualitarismo-elites.pdf

Rousseau, J. J. (1987). Discurso sobre el origen y los fundamentos de la desigualdad entre los hombres y otros escritos. Madrid, España: Tecnos.

Saborit, P. (2006). Vidas adosadas. El miedo a los semejantes en la sociedad contemporánea. Barcelona, España: Anagrama.

Spaemann, R. (2010). Personas. Acerca de la distinción entre "algo" y "alguien" (2. ${ }^{a}$ ed.). Navarra, España: Ediciones Universidad de Navarra.

Supiot, A. (2007). Homo juridicus. Ensayo sobre la función antropológica del derecho. Buenos Aires, Argentina: Siglo XXI.

Torres Vásquez, A. (2011). Introducción al derecho. Teoría general del derecho (4. ${ }^{a}$ ed.). Lima, Perú: IDEMSA.

Vincent-Thomas, L. (setiembre, 2007). Muerte física y muerte espiritual. La Gaceta del Fondo de Cultura Económica, 441. Recuperado de http://www.fondodeculturaeconomica.com/subdirectorios_site/ gacetas/sep_2007.pdf

White, T. I. (2010). Ethical implications of dolphin intelligence: Dolphins as nonhuman persons. Ponencia presentada en la Reunión Anual de la American Association for the Advancement of Science. San Diego, CA. 